\title{
Research Article \\ Jump Telegraph Processes and Financial Markets with Memory
}

Nikita Ratanov

Received 21 November 2006; Revised 22 April 2007; Accepted 9 August 2007

The paper develops a new class of financial market models. These models are based on generalized telegraph processes with alternating velocities and jumps occurring at switching velocities. The model under consideration is arbitrage-free and complete if the directions of jumps in stock prices are in a certain correspondence with their velocity and with the behaviour of the interest rate. A risk-neutral measure and arbitrage-free formulae for a standard call option are constructed. This model has some features of models with memory, but it is more simple.

Copyright $\odot 2007$ Nikita Ratanov. This is an open access article distributed under the Creative Commons Attribution License, which permits unrestricted use, distribution, and reproduction in any medium, provided the original work is properly cited.

\section{Introduction}

It is widely recognized that the dynamics of asset returns cannot be adequately described by geometric Brownian motion with constant volatility. Due to the market efficiency, alternative models are based on random processes with independent increments (Brownian motion, jump diffusions, and the variance gamma process). The development of non-semimartingale models is focused mainly on accounting for the dependence of asset prices on the past (long-term memory processes, fractional Brownian motion, etc.). However, till now there is still no commonly accepted theory on this topic, nor adequate uses of existing theoretical results in practice (see, e.g., [1]).

On the other hand, models which are based on pure jump processes with independent increments recently were widely proposed (see, e.g., [2-4]). Carr et al. [2] empirically show that the diffusion component could be ignored, if the pure jump process allows infinite activity. This means that there are infinitely many small jumps which asymptotically model a diffusion component. Usually these models are incomplete. 
This paper proposes a different model. As a basis for building, we take a pure jump process $(\sigma(t), t \geq 0)$ with values \pm 1 and (finite) transition probability intensities $\lambda_{ \pm}$. Let $c_{ \pm}, r_{ \pm}, h_{ \pm}$be real numbers such that $c_{+}>c_{-}, r_{ \pm}>0$, and $h_{ \pm}>-1$. We introduce the processes $\left(c_{\sigma(t)}, t \geq 0\right),\left(h_{\sigma(t)}, t \geq 0\right)$, and $\left(r_{\sigma(t)}, t \geq 0\right)$ and we define $X_{s}=\left(X_{s}(t), t \geq 0\right)$ by $X_{s}(t)=\int_{0}^{t} c_{\sigma(\tau)} \mathrm{d} \tau$ and a pure jump process $J_{s}=\left(J_{s}(t), t \geq 0\right)$ with alternating jumps of sizes $h_{ \pm}$. The evolution of the risky asset $S(t)$ is determined by a stochastic exponential of the sum $X_{s}+J_{s}$. The risk-free asset is given by the usual exponential of the process $Y_{s}=$ $\left(Y_{s}(t), t \geq 0\right)=\left(\int_{0}^{t} r_{\sigma(\tau)} \mathrm{d} \tau, t \geq 0\right)$. Here and below the subscript $s$ indicates the starting value $s=\sigma(0)$ of $\sigma(t)$.

In view of such trajectories, the market is set up as a continuous process that evolves with velocities $c_{+}$or $c_{-}$, changes the direction of movement from $c_{ \pm}$to $c_{\mp}$, and exhibits jumps of sizes $h_{ \pm}$whenever velocity changes. The different parameters for up and down movements in particular lead to a gain/loss asymmetry.

The interest rate in the market is stochastic with the values $r_{ \pm}$such that $\left(c_{ \pm}-r_{ \pm}\right) h_{ \pm}<0$ which means that the current trend of discounted prices and the direction of the next price jump should be opposite. The processes $X_{s}, s= \pm$ are defined by the pair of states $\left(c_{ \pm}, \lambda_{ \pm}\right)$and are called telegraph processes with states $\left(c_{ \pm}, \lambda_{ \pm}\right)$. They describe continuous price trends (upward or downward) between random instants. Changes in these trends are accompanied by jumps of sizes $h_{ \pm}$. Our model uses parameters $c_{ \pm}$to capture bullish and bearish trends in a market evolution, and values $h_{ \pm}$to describe sizes of possible jumps and spikes. Thus, we study a model that is both realistic and general enough to enable us to incorporate different trends and extreme events. This model describes adequately the processes on oversold and overbought markets, when changes on the market tendencies accumulate in the course of time.

Sections 2 and 3 deal with the properties of such processes and the mathematical model of the market. Among the relevant results, we construct a unique martingale measure based on Girsanov's theorem. This measure guarantees the absence of arbitrage in our setting and shows that, under some scaling normalization, our model converges to that of Black-Scholes in distribution. The final short section (Section 4) explains memory features of the proposed model in terms of historical volatility.

Telegraph processes have been studied before in different probabilistic and financial aspects (see, e.g., [5-8]). These processes have been exploited for stochastic volatility modeling [9], in actuarial problems [10], as well as for obtaining a "telegraph analog" of the Black-Scholes model (see Di Crescenzo and Pellerey [11]). In contrast with the latter paper by Di Crescenzo and Pellerey, we use more complicated and delicate construction of such a model to avoid arbitrage and to develop an adequate option pricing theory in this framework.

\section{Jump telegraph processes}

Let $(\Omega, \mathscr{F}, \mathbb{P})$ be a complete probability space, and let $\lambda_{ \pm}$be positive numbers. We consider two counting Poisson processes $N_{+}=\left(N_{+}(t), t \geq 0\right)$ and $N_{-}=\left(N_{-}(t), t \geq 0\right)$ with alternating intensities $\lambda_{+}, \lambda_{-}, \lambda_{+}, \ldots$ and $\lambda_{-}, \lambda_{+}, \lambda_{-}, \ldots$, respectively, that is, as $\Delta t \rightarrow 0$

$$
\mathbb{P}\left(N_{+}(t+\Delta t)=2 n+1 \mid N_{+}(t)=2 n\right)=\lambda_{+} \Delta t+o(\Delta t)
$$




$$
\begin{array}{r}
\mathbb{P}\left(N_{+}(t+\Delta t)=2 n+2 \mid N_{+}(t)=2 n+1\right)=\lambda_{-} \Delta t+o(\Delta t), \\
\mathbb{P}\left(N_{-}(t+\Delta t)=2 n+1 \mid N_{-}(t)=2 n\right)=\lambda_{-} \Delta t+o(\Delta t), \\
\mathbb{P}\left(N_{-}(t+\Delta t)=2 n+2 \mid N_{-}(t)=2 n+1\right)=\lambda_{+} \Delta t+o(\Delta t), \\
n=0,1,2, \ldots .
\end{array}
$$

Further we will consider all stochastic processes subscribed by + or - to be adapted to the filtrations $\mathbf{F}^{+}=\left(\mathbf{F}_{t}^{+}\right)_{t \geq 0}$ and $\mathbf{F}^{-}=\left(\mathbf{F}_{t}^{-}\right)_{t \geq 0}$ generated by $N_{+}$and $N_{-}$, respectively. We denote $\sigma_{+}(t)=(-1)^{N_{+}(t)}$ and $\sigma_{-}(t)=-(-1)^{N_{-}(t)}$.

Let $h_{ \pm} \in(-1, \infty)$ and $c_{ \pm}$be real numbers. Consider the (right continuous) processes

$$
\begin{array}{rlrl}
X_{+}(t) & =\int_{0}^{t} c_{\sigma_{+}(\tau)} \mathrm{d} \tau, & J_{+}(t)=\int_{0}^{t} h_{\sigma_{+}(\tau)} \mathrm{d} N_{+}(\tau), \\
X_{-}(t)=\int_{0}^{t} c_{\sigma_{-}(\tau)} \mathrm{d} \tau, & J_{-}(t)=\int_{0}^{t} h_{\sigma_{-}(\tau)} \mathrm{d} N_{-}(\tau) .
\end{array}
$$

The subscripts \pm indicate the initial state of the processes.

Introducing the jumping times $\tau_{1}, \tau_{2}, \ldots$ of the processes $N_{ \pm}$and setting $\tau_{0}=0$, we have the following representation (e.g., for the subscript + ):

$$
X_{+}(t)=\sum_{j=1}^{N_{+}(t)} c_{\sigma_{+}\left(t_{j}-\right)}\left(\tau_{j}-\tau_{j-1}\right)+c_{\sigma_{+}(t)}\left(t-\tau_{N_{+}(t)}\right), \quad J_{+}(t)=\sum_{j=1}^{N_{+}(t)} h_{\sigma_{+}\left(\tau_{j}-\right)} .
$$

The processes $X_{ \pm}=\left(X_{ \pm}(t), t \geq 0\right)$ are usually referred to as (integrated) telegraph process (see Goldstein [5] and Kac [12,6]). The processes $J_{ \pm}=\left(J_{ \pm}(t), t \geq 0\right)$ are pure jump processes with alternating jump sizes $h_{ \pm}$. Let us introduce the standard telegraph and jump processes associated with $\mathcal{c}_{ \pm}= \pm 1$ and $h_{ \pm}= \pm 1$ :

$$
\begin{array}{ll}
X_{+}^{0}(t)=\int_{0}^{t} \sigma_{+}(\tau) \mathrm{d} \tau, & J_{+}^{0}(t)=\mathbf{1}_{\left\{N_{+}(t) \text { is odd }\right\}}=\frac{1-\sigma_{+}(t)}{2}, \\
X_{-}^{0}(t)=\int_{0}^{t} \sigma_{-}(\tau) \mathrm{d} \tau, & J_{-}^{0}(t)=-\mathbf{1}_{\left\{N_{+}(t) \text { is odd }\right\}}=\frac{-1-\sigma_{-}(t)}{2} .
\end{array}
$$

Proposition 2.1. The processes $X_{ \pm}$and $J_{ \pm}$are linearly connected with $X_{ \pm}^{0}$ and $J_{ \pm}^{0}$ :

$$
X_{ \pm}(t)=a X_{ \pm}^{0}(t)+A t, \quad J_{ \pm}(t)=b J_{ \pm}^{0}(t)+B N_{ \pm}(t)
$$

where $A=\left(c_{+}+c_{-}\right) / 2, a=\left(c_{+}-c_{-}\right) / 2, B=\left(h_{+}+h_{-}\right) / 2$, and $b=\left(h_{+}-h_{-}\right) / 2$. 
4 Journal of Applied Mathematics and Stochastic Analysis

Proof. We only consider the case related to the subscript + . The other case is quite similar. We have

$$
\begin{aligned}
X_{+}(t) & =c_{+} \int_{0}^{t} 1_{\left\{N_{+}(\tau) \text { is even }\right\}} \mathrm{d} \tau+c_{-} \int_{0}^{t} \mathbf{1}_{\left\{N_{+}(\tau) \text { is odd }\right\}} \mathrm{d} \tau \\
& =c_{+} t-\left(c_{+}-c_{-}\right) \int_{0}^{t} \mathbf{1}_{\left\{N_{+}(\tau) \text { is odd }\right\}} \mathrm{d} \tau \\
& =(A+a) t-2 a \int_{0}^{t} \mathbf{1}_{\left\{N_{+}(\tau) \text { is odd }\right\}} \mathrm{d} \tau .
\end{aligned}
$$

For $c_{ \pm}= \pm 1$, we find that $X_{+}^{0}(t)=t-2 \int_{0}^{t} \mathbf{1}_{\left\{N_{+}(\tau) \text { is odd }\right\}} \mathrm{d} \tau$. As a byproduct, $X_{+}(t)=A t+$ $a X_{+}^{0}(t)$.

On the other hand,

$$
\begin{aligned}
J_{+}(t)= & \sum_{j=1}^{N_{+}(t)} h_{\sigma_{+}\left(\tau_{j}-\right)} \\
= & \begin{cases}\left(h_{+}+h_{-}\right) \frac{N_{+}(t)}{2}=B N_{+}(t), & \text { if } N_{+}(t) \text { is even, } \\
\left(h_{+}+h_{-}\right) \frac{N_{+}(t)-1}{2}+h_{+}=B N_{+}(t)+b, & \text { if } N_{+}(t) \text { is odd, }\end{cases} \\
=B N_{+}(t)+b \mathbf{1}_{\left\{N_{+}(t) \text { is odd }\right\}}=B N_{+}(t)+b J_{+}^{0}(t) . &
\end{aligned}
$$

The next theorem could be considered as a version of the Doob-Meyer decomposition for telegraph processes.

Theorem 2.2. The jump telegraph processes $Z_{+}:=X_{+}+J_{+}$and $Z_{-}:=X_{-}+J_{-}$are martingales if and only if $c_{+}=-\lambda_{+} h_{+}$and $c_{-}=-\lambda_{-} h_{-}$.

The proof is based on direct calculations of the conditional expectations $\mathbb{E}\left(X_{ \pm}(t)+\right.$ $J_{ \pm}(t) \mid \mathbf{F}_{s}^{ \pm}$) (see Remark 2.10 below).

We can obtain the exact distribution of jump telegraph processes $Z_{ \pm}=X_{ \pm}+J_{ \pm}$in terms of generalized probability densities $p_{ \pm}(x, t)$, which are defined by

$$
\begin{aligned}
& \mathbb{P}\left(X_{+}(t)+J_{+}(t) \in \Delta\right)=\int_{\Delta} p_{+}(x, t) d x \\
& \mathbb{P}\left(X_{-}(t)+J_{-}(t) \in \Delta\right)=\int_{\Delta} p_{-}(x, t) d x
\end{aligned}
$$

for any Borelian set $\Delta$. By generalized densities we mean that the distributions of $X_{ \pm}+J_{ \pm}$ are made up of an absolutely continuous part (i.e., a genuine density) and a discrete part. 
Theorem 2.3. Functions $p_{ \pm}$solve the system

$$
\begin{aligned}
& \frac{\partial p_{+}}{\partial t}(x, t)+c_{+} \frac{\partial p_{+}}{\partial x}(x, t)=-\lambda_{+}\left[p_{+}(x, t)-p_{-}\left(x-h_{+}, t\right)\right] \\
& \frac{\partial p_{-}}{\partial t}(x, t)+c_{-} \frac{\partial p_{-}}{\partial x}(x, t)=-\lambda_{-}\left[p_{-}(x, t)-p_{+}\left(x-h_{-}, t\right)\right]
\end{aligned}
$$

with initial condition $p_{ \pm}(x, 0)=\delta(x)$.

Proof. First notice that from the properties of counting Poisson process (see, e.g., [13]) for $t_{2}>t_{1} \geq 0$ it follows that

$$
Z_{ \pm}\left(t_{2}\right)=Z_{ \pm}\left(t_{1}\right)+\int_{t_{1}}^{t_{2}} c_{\sigma_{ \pm}(\tau)} \mathrm{d} \tau+\int_{t_{1}}^{t_{2}} h_{\sigma_{ \pm}(\tau)} \mathrm{d} N_{ \pm}(\tau)=Z_{ \pm}\left(t_{1}\right)+Z_{\sigma_{ \pm}\left(t_{1}\right)}^{\prime}\left(t_{2}-t_{1}\right)
$$

where $Z_{ \pm}^{\prime}$ are copies of the processes $Z_{ \pm}$which are independent of $Z_{ \pm}$.

Next, notice that $Z_{ \pm}(\Delta t)=c_{ \pm} \Delta t$, if $N_{ \pm}(\Delta t)=0$, and $Z_{ \pm}(\Delta t)=c_{ \pm} \Delta t+h_{ \pm}+o(\Delta t), \Delta t \rightarrow$ 0 , if $N_{ \pm}(\Delta t)=1$. Moreover,

$$
N_{ \pm}(\Delta t)= \begin{cases}0 & \text { with probability } 1-\lambda_{ \pm} \Delta t+o(\Delta t), \\ 1 & \text { with probability } \lambda_{ \pm} \Delta t+o(\Delta t), \quad \Delta t \longrightarrow 0 . \\ \geq 2 & \text { with probability } o(\Delta t)\end{cases}
$$

Applying (2.10) with the choice $t_{1}=\Delta t, t_{2}=t+\Delta t$ we have

$$
\begin{aligned}
p_{+}(x, t & +\Delta t) \\
= & \frac{\mathbb{P}\left\{Z_{+}(t+\Delta t) \in \mathrm{d} x\right\}}{\mathrm{d} x} \\
= & \left(1-\lambda_{+} \Delta t\right) \frac{\mathbb{P}\left\{Z_{+}(t) \in \mathrm{d} x-c_{+} \Delta t\right\}}{\mathrm{d} x} \\
& +\lambda_{+} \Delta t \frac{\mathbb{P}\left\{Z_{-}(t) \in \mathrm{d} x-h_{+}-c_{+} \Delta t-o(\Delta t)\right\}}{\mathrm{d} x}+o(\Delta t) \\
= & \left(1-\lambda_{+} \Delta t\right) p_{+}\left(x-c_{+} \Delta t, t\right)+\lambda_{+} \Delta t p_{-}\left(x-h_{+}-c_{+} \Delta t-o(\Delta t), t\right)+o(\Delta t),
\end{aligned}
$$

which immediately implies the first equation of (2.9). The second equation can be obtained similarly.

Remark 2.4. Applying Kac's trick (see [12]), it is easy to prove that in the particular case $c_{+}=+1, c_{-}=-1, \lambda_{-}=\lambda_{+}:=\lambda$, and $h_{ \pm}=0$ the densities $p_{ \pm}(x, t)$ satisfy the so-called telegraph equation

$$
\frac{\partial^{2} u}{\partial t^{2}}+2 \lambda \frac{\partial u}{\partial t}=\frac{\partial^{2} u}{\partial x^{2}}
$$

which is a damped wave equation. 
6 Journal of Applied Mathematics and Stochastic Analysis

Conditioning on the number of switches one can show that system (2.9) has the following solution:

$$
p_{ \pm}(x, t)=\mathrm{e}^{-\Lambda_{a} t-\lambda_{a} x} \sum_{n=0}^{\infty} q_{ \pm}^{(n)}\left(x-j_{ \pm}^{(n)}, t\right)
$$

where

$$
\begin{gathered}
\lambda_{a}=\frac{\lambda_{+}-\lambda_{-}}{2 a}, \quad \Lambda_{a}=\lambda_{ \pm}-\lambda_{a} c_{ \pm}=\frac{c_{+} \lambda_{-}-c_{-} \lambda_{+}}{2 a}, \quad 2 a=c_{+}-c_{-}, \\
j_{ \pm}^{(n)}= \begin{cases}n B, & n=2 k, \\
(n-1) B+h_{ \pm}, & n=2 k+1,\end{cases}
\end{gathered}
$$

Here $q_{ \pm}^{(0)}(x, t)=\delta\left(x-c_{ \pm} t\right)$ and, setting $\theta(x, t)=\mathbf{1}_{\left\{c_{-} t<x<c_{+} t\right\}}$, for $n \geq 1$

$$
\begin{aligned}
& q_{+}^{(2 n)}(x, t)=\frac{\lambda_{+}^{n} \lambda_{-}^{n}}{(2 a)^{2 n}} \cdot \frac{\left(c_{+} t-x\right)^{n-1}\left(x-c_{-} t\right)^{n}}{(n-1) ! n !} \theta(x, t), \\
& q_{-}^{(2 n)}(x, t)=\frac{\lambda_{+}^{n} \lambda_{-}^{n}}{(2 a)^{2 n}} \cdot \frac{\left(c_{+} t-x\right)^{n}\left(x-c_{-} t\right)^{n-1}}{n !(n-1) !} \theta(x, t),
\end{aligned}
$$

and for $n \geq 0$

$$
\begin{aligned}
& q_{+}^{(2 n+1)}(x, t)=\frac{\lambda_{+}^{n+1} \lambda_{-}^{n}}{(2 a)^{2 n+1}} \cdot \frac{\left(c_{+} t-x\right)^{n}\left(x-c_{-} t\right)^{n}}{(n !)^{2}} \theta(x, t), \\
& q_{-}^{(2 n+1)}(x, t)=\frac{\lambda_{+}^{n} \lambda_{-}^{n+1}}{(2 a)^{2 n+1}} \cdot \frac{\left(c_{+} t-x\right)^{n}\left(x-c_{-} t\right)^{n}}{(n !)^{2}} \theta(x, t) .
\end{aligned}
$$

Alternatively one can obtain formulae (2.14)-(2.17) by applying the results of Zacks [8]. There, the probability densities $p_{ \pm}(x, t)$ are expressed in terms of Poisson and Erlang densities.

Formulae (2.14)-(2.17) give the following rules of changes in the intensities $\lambda_{ \pm}$: if $\lambda_{+}$ is changed to $\lambda_{+}^{\prime}$ and $\lambda_{-}$is changed to $\lambda_{-}^{\prime}$, the probability densities $p_{ \pm}$will be changed to

$$
p_{ \pm}^{\prime}(x, t)=\mathrm{e}^{-\Lambda_{a}^{\prime} t-\lambda_{a}^{\prime} x} \sum_{n=0}^{\infty} q_{ \pm}^{\prime(n)}\left(x-j_{ \pm}^{(n)}, t\right)
$$

where $\lambda_{a}^{\prime}=\left(\lambda_{+}^{\prime}-\lambda_{-}^{\prime}\right) / 2 a, \Lambda_{a}^{\prime}=\left(c_{+} \lambda_{-}^{\prime}-c_{-} \lambda_{+}^{\prime}\right) / 2 a$, and $q_{ \pm}^{\prime(n)}(x, t)=q_{ \pm}^{(n)}(x, t) \times \kappa_{\lambda^{\prime} / \lambda, \pm}^{(n)}$ with

$$
\begin{aligned}
& \kappa_{\lambda^{\prime} / \lambda, \pm}^{(2 n)}=\left(\frac{\lambda_{+}^{\prime}}{\lambda_{+}}\right)^{n}\left(\frac{\lambda_{-}^{\prime}}{\lambda_{-}}\right)^{n}, \\
& \kappa_{\lambda^{\prime} / \lambda,+}^{(2 n+1)}=\left(\frac{\lambda_{+}^{\prime}}{\lambda_{+}}\right)^{n+1}\left(\frac{\lambda_{-}^{\prime}}{\lambda_{-}}\right)^{n}, \quad n=0,1, \ldots \\
& \kappa_{\lambda^{\prime} / \lambda,-}^{(2 n+1)}=\left(\frac{\lambda_{+}^{\prime}}{\lambda_{+}}\right)^{n}\left(\frac{\lambda_{-}^{\prime}}{\lambda_{-}}\right)^{n+1},
\end{aligned}
$$


Remark 2.5. In particular case, if $B=h_{+}+h_{-}=0$, then formulae (2.14)-(2.17) become

$$
\begin{aligned}
p_{ \pm}(x, t)=\mathrm{e}^{-\lambda_{ \pm} t} \cdot \delta\left(x-c_{ \pm} t\right) & \\
+\frac{\mathrm{e}^{-\Lambda t-\lambda x}}{2 a}[ & \lambda_{ \pm} I_{0}\left(\sqrt{\lambda_{+} \lambda_{-}\left(c_{+} t-x+h_{ \pm}\right)\left(x-h_{ \pm}-c_{-} t\right)} / a\right) \theta\left(x-h_{ \pm}, t\right) \\
& \left.+\sqrt{\lambda_{+} \lambda_{-}}\left(\frac{c_{+} t-x}{x-c_{-} t}\right)^{\mp 1 / 2} I_{1}\left(\sqrt{\lambda_{+} \lambda_{-}\left(c_{+} t-x\right)\left(x-c_{-} t\right)} / a\right) \theta(x, t)\right]
\end{aligned}
$$

where $I_{0}(z)=\sum_{n=0}^{\infty}(z / 2)^{2 n} /(n !)^{2}$ and $I_{1}(z)=I_{0}^{\prime}(z)$ are usual modified Bessel functions. Compare with [14].

Using (2.9) one can deduce equations for $\mathbb{E}\left[f\left(X_{ \pm}(t)+J_{ \pm}(t)\right)\right]$. More precisely, we have the following corollary.

Corollary 2.6. Let $f=(f(x), x \in \mathbb{R})$ and $\alpha_{ \pm}=\left(\alpha_{ \pm}(t), t \geq 0\right)$ be smooth deterministic functions. Then

$$
u_{ \pm}(x, t)=\mathbb{E}\left[f\left(x-\alpha_{ \pm}(t)+X_{ \pm}(t)+J_{ \pm}(t)\right)\right]
$$

form a solution of the system

$$
\begin{aligned}
& \frac{\partial u_{+}}{\partial t}(x, t)-\left(c_{+}-\dot{\alpha}_{+}(t)\right) \frac{\partial u_{+}}{\partial x}(x, t)=-\lambda_{+}\left[u_{+}(x, t)-u_{-}\left(x+\beta_{+}(t), t\right)\right], \\
& \frac{\partial u_{-}}{\partial t}(x, t)-\left(c_{-}-\dot{\alpha}_{-}(t)\right) \frac{\partial u_{-}}{\partial x}(x, t)=-\lambda_{-}\left[u_{-}(x, t)-u_{+}\left(x+\beta_{-}(t), t\right)\right]
\end{aligned}
$$

with $\beta_{+}(t)=h_{+}-\left(\alpha_{+}(t)-\alpha_{-}(t)\right), \beta_{-}(t)=h_{-}-\left(\alpha_{-}(t)-\alpha_{+}(t)\right)$, and $\dot{\alpha}_{ \pm}=\mathrm{d} \alpha_{ \pm} / \mathrm{d} t$.

Proof. Notice that by definition $u_{ \pm}(x, t)=\int_{-\infty}^{\infty} f\left(x-\alpha_{ \pm}(t)+y\right) p_{ \pm}(y, t) \mathrm{d} y$. Hence

$$
\frac{\partial u_{ \pm}}{\partial t}(x, t)=\int_{-\infty}^{\infty} f\left(x-\alpha_{ \pm}(t)+y\right) \frac{\partial p_{ \pm}}{\partial t}(y, t) \mathrm{d} y-\dot{\alpha}_{ \pm}(t) \frac{\partial u_{ \pm}}{\partial x}(x, t) .
$$

Applying (2.9) immediately yields (2.22).

We apply (2.22) to deduce formulae for the mean value and the variance of the jump telegraph process:

$$
m_{ \pm}(t)=\mathbb{E}\left(X_{ \pm}(t)+J_{ \pm}(t)\right), \quad s_{ \pm}(t)=\operatorname{Var}\left(X_{ \pm}(t)+J_{ \pm}(t)\right) .
$$

Seeking simplicity it will be done only in the symmetric case.

Theorem 2.7. Suppose $\lambda_{-}=\lambda_{+}:=\lambda$ and set $\gamma_{+}=-2 a\left(a / \lambda+h_{+}\right), \gamma_{-}=-2 a\left(a / \lambda-h_{-}\right)$, and $\Phi_{\lambda}(t)=\left(1-\mathrm{e}^{-2 \lambda t}\right) /(2 \lambda t)$. Then

$$
\begin{aligned}
m_{ \pm}(t) & =\left[A+\lambda B \pm(a+\lambda b) \Phi_{\lambda}(t)\right] t \\
s_{ \pm}(t) & =\left[\frac{a^{2}}{\lambda}+\lambda B^{2}+\frac{(a+\lambda b)^{2} \Phi_{2 \lambda}(t)}{\lambda}+\gamma_{ \pm} \Phi_{\lambda}(t) \pm 2 B(a+\lambda b) \mathrm{e}^{-2 \lambda t}\right] t .
\end{aligned}
$$


8 Journal of Applied Mathematics and Stochastic Analysis

Proof. First, we apply Corollary 2.6 with the choices $f(x)=x$ and $\alpha_{ \pm}(t)=0$. Then $\beta_{ \pm}(t)$ $=h_{ \pm}$and $u_{ \pm}(x, t)=\mathbb{E}\left(x+X_{ \pm}(t)+J_{ \pm}(t)\right)=x+m_{ \pm}(t)$. We obviously have $\left(\partial u_{ \pm} / \partial x\right)(x, t)=$ $1,\left(\partial u_{ \pm} / \partial t\right)(x, t)=\left(\mathrm{d} m_{ \pm} / \mathrm{d} t\right)(t)$, and $u_{ \pm}(x, t)-u_{\mp}\left(x+\beta_{ \pm}(t), t\right)=m_{ \pm}(t)-m_{\mp}(t)-h_{ \pm}$. By (2.22) we obtain the following system for $m_{ \pm}$:

$$
\begin{aligned}
& \frac{d m_{+}}{d t}=-\lambda\left(m_{+}-m_{-}\right)+c_{+}+\lambda h_{+}, \\
& \frac{d m_{-}}{d t}=-\lambda\left(m_{-}-m_{+}\right)+c_{-}+\lambda h_{-}
\end{aligned}
$$

with zero initial conditions.

Now, with the choices $f(x)=x^{2}$ and $\alpha_{ \pm}(t)=m_{ \pm}(t)$, we have $\beta_{ \pm}(t)=h_{ \pm}-\left(m_{ \pm}(t)-\right.$ $\left.m_{\mp}(t)\right)$ and $u_{ \pm}(x, t)=\mathbb{E}\left[\left(x-m_{ \pm}(t)+X_{ \pm}(t)+J_{ \pm}(t)\right)^{2}\right]=x^{2}+s_{ \pm}(t)$. Therefore, $\left(\partial u_{ \pm} / \partial x\right)(x, t)=2 x,\left(\partial u_{ \pm} / \partial t\right)(x, t)=\left(\mathrm{d} s_{ \pm} / \mathrm{d} t\right)(t)$, and $u_{ \pm}(x, t)-u_{\mp}\left(x+\beta_{ \pm}(t), t\right)=s_{ \pm}(t)-$ $s_{\mp}(t)-2 \beta_{ \pm}(t) x-\beta_{ \pm}(t)^{2}$. Putting this into (2.22) we get

$$
\begin{aligned}
\frac{\mathrm{d} s_{ \pm}}{\mathrm{d} t}(t) & =2 x\left(c_{ \pm}+\lambda \beta_{ \pm}(t)-\frac{\mathrm{d} m_{ \pm}}{\mathrm{d} t}(t)\right)-\lambda\left(s_{ \pm}(t)-s_{\overline{+}}(t)\right)+\lambda \beta_{ \pm}(t)^{2} \\
& =-\lambda\left(s_{ \pm}(t)-s_{\bar{\mp}}(t)\right)+\lambda\left(h_{ \pm}-\left(m_{ \pm}(t)-m_{\mp}(t)\right)\right)^{2}
\end{aligned}
$$

since by $(2.27) c_{ \pm}+\lambda \beta_{ \pm}(t)-\left(\mathrm{d} m_{ \pm} / \mathrm{d} t\right)(t)=0$. This yields the following system for $s_{ \pm}$:

$$
\begin{aligned}
& \frac{d s_{+}}{d t}=-\lambda\left(s_{+}-s_{-}\right)+\lambda\left(h_{+}+m_{-}-m_{+}\right)^{2}, \\
& \frac{d s_{-}}{d t}=-\lambda\left(s_{-}-s_{+}\right)+\lambda\left(h_{-}+m_{+}-m_{-}\right)^{2}
\end{aligned}
$$

with zero initial conditions.

Systems (2.27) and (2.29) can be rewritten in a matrix form. Setting $\Lambda=\left(\begin{array}{cc}-\lambda & \lambda \\ \lambda & -\lambda\end{array}\right)$, $\mathbf{m}=\left(\begin{array}{c}m_{+} \\ m_{-}\end{array}\right), \mathbf{s}=\left(\begin{array}{c}s_{+} \\ s_{-}\end{array}\right), \mathbf{k}=\left(\begin{array}{c}c_{+}+\lambda h_{+} \\ c_{-}+\lambda h_{-}\end{array}\right), \mathbf{l}=\left(\begin{array}{l}\lambda\left(h_{+}+m_{-}-m_{+}\right)^{2} \\ \lambda\left(h_{-}+m_{+}-m_{-}\right)^{2}\end{array}\right)$, we have

$$
\frac{\mathrm{d} \mathbf{m}}{\mathrm{d} t}(t)=\Lambda \mathbf{m}(t)+\mathbf{k}, \quad \frac{\mathrm{d} \mathbf{s}}{\mathrm{d} t}(t)=\Lambda \mathbf{s}(t)+\mathbf{l}(t) .
$$

Hence

$$
\mathbf{m}(t)=\int_{0}^{t} \mathrm{e}^{(t-\tau) \Lambda} \mathbf{k} \mathrm{d} \tau, \quad \mathbf{s}(t)=\lambda \int_{0}^{t} \mathrm{e}^{(t-\tau) \Lambda} \mathbf{l}(\tau) \mathrm{d} \tau .
$$

Observing the identity $\Lambda^{2}=-2 \lambda \Lambda$ which implies $\Lambda^{n}=(-2 \lambda)^{n-1} \Lambda$ for any $n \geq 1$, we deduce $\mathrm{e}^{t \Lambda}=I+\sum_{n=1}^{\infty}\left((-2 \lambda)^{n-1} t^{n} / n !\right) \Lambda=I+(1 / 2 \lambda)\left(1-\mathrm{e}^{-2 \lambda t}\right) \Lambda$ and then

$$
\int_{0}^{t} \mathrm{e}^{(t-\tau) \Lambda} \mathrm{d} \tau=t I+\frac{1}{2 \lambda}\left(t-\frac{1}{2 \lambda}\left(1-\mathrm{e}^{-2 \lambda t}\right)\right) \Lambda=t\left[I+\frac{1}{2 \lambda}\left(1-\Phi_{\lambda}(t)\right) \Lambda\right] .
$$


As a result, we get $\mathbf{m}(t)=t\left[(I+(1 / 2 \lambda) \Lambda) \mathbf{k}-(1 / 2 \lambda) \Phi_{\lambda}(t) \Lambda \mathbf{k}\right]$, where

$$
\begin{gathered}
\Lambda \mathbf{k}=\left(c_{+}-c_{-}+\lambda\left(h_{+}-h_{-}\right)\right)\left(\begin{array}{c}
-\lambda \\
\lambda
\end{array}\right)=(a+\lambda b)\left(\begin{array}{c}
-2 \lambda \\
2 \lambda
\end{array}\right) \\
\left(I+\frac{1}{2 \lambda} \Lambda\right) \mathbf{k}=\frac{1}{2}\left(\begin{array}{ll}
1 & 1 \\
1 & 1
\end{array}\right)\left(\begin{array}{l}
c_{+}+\lambda h_{+} \\
c_{-}+\lambda h_{-}
\end{array}\right)=\frac{1}{2}\left(c_{+}+c_{-}+\lambda\left(h_{+}+h_{-}\right)\right)\left(\begin{array}{l}
1 \\
1
\end{array}\right)=(A+\lambda B)\left(\begin{array}{l}
1 \\
1
\end{array}\right) .
\end{gathered}
$$

Thus $\mathbf{m}(t)=t\left(\begin{array}{c}(A+\lambda B)+(a+\lambda b) \Phi_{\lambda}(t) \\ (A+\lambda B)-(a+\lambda b) \Phi_{\lambda}(t)\end{array}\right)$, from which $(2.25)$ emerges.

Next, in order to determine $\mathbf{s}$, we note that $m_{+}(t)-m_{-}(t)=2(a+\lambda b) \Phi_{\lambda}(t)$ and then

$$
\mathbf{l}(\tau)=\lambda\left(\begin{array}{c}
h_{+}^{2} \\
h_{-}^{2}
\end{array}\right)+4 \lambda(a+\lambda b) \tau \Phi_{\lambda}(\tau)\left(\begin{array}{c}
-h_{+} \\
h_{-}
\end{array}\right)+4 \lambda(a+\lambda b)^{2} \tau^{2} \Phi_{\lambda}(\tau)^{2}\left(\begin{array}{l}
1 \\
1
\end{array}\right)
$$

Putting this expression into (2.31), we see that we need to evaluate the integrals $\int_{0}^{t}(2 \lambda \tau) \Phi_{\lambda}(\tau) \mathrm{e}^{(t-\tau) \Lambda} \mathrm{d} \tau$ and $\int_{0}^{t}(2 \lambda \tau)^{2} \Phi_{\lambda}(\tau)^{2} \mathrm{e}^{(t-\tau) \Lambda}\left(\begin{array}{l}1 \\ 1\end{array}\right) \mathrm{d} \tau$.

First, we have

$$
\begin{aligned}
\int_{0}^{t}(2 \lambda \tau) & \Phi_{\lambda}(\tau) \mathrm{e}^{(t-\tau) \Lambda} \mathrm{d} \tau \\
= & \int_{0}^{t}\left(1-\mathrm{e}^{-2 \lambda \tau}\right)\left(I+\frac{1}{2 \lambda}\left(1-\mathrm{e}^{-2 \lambda(t-\tau)}\right) \Lambda\right) \mathrm{d} \tau \\
= & {\left[\int_{0}^{t}\left(1-\mathrm{e}^{-2 \lambda \tau}\right) \mathrm{d} \tau\right]\left(I+\frac{1}{2 \lambda} \Lambda\right)-\frac{1}{2 \lambda}\left[\int_{0}^{t}\left(\mathrm{e}^{-2 \lambda(t-\tau)}-\mathrm{e}^{-2 \lambda t}\right) \mathrm{d} \tau\right] \Lambda } \\
= & \left(t-\frac{1}{2 \lambda}\left(1-\mathrm{e}^{-2 \lambda t}\right)\right)\left(I+\frac{1}{2 \lambda} \Lambda\right)-\frac{1}{2 \lambda}\left(\frac{1}{2 \lambda}\left(1-\mathrm{e}^{-2 \lambda t}\right)-t \mathrm{e}^{-2 \lambda t}\right) \Lambda \\
= & t\left(1-\Phi_{\lambda}(t)\right) I+\frac{t}{\lambda}\left(1-(1+\lambda t) \Phi_{\lambda}(t)\right) \Lambda
\end{aligned}
$$

Second, we have, since $\Lambda\left(\begin{array}{l}1 \\ 1\end{array}\right)=\left(\begin{array}{l}0 \\ 0\end{array}\right)$ and then $\mathrm{e}^{(t-\tau) \Lambda}\left(\begin{array}{l}1 \\ 1\end{array}\right)=\left(\begin{array}{l}1 \\ 1\end{array}\right)$,

$$
\begin{aligned}
\int_{0}^{t}(2 \lambda \tau)^{2} \Phi_{\lambda}(\tau)^{2} \mathrm{e}^{(t-\tau) \Lambda}\left(\begin{array}{l}
1 \\
1
\end{array}\right) \mathrm{d} \tau & =\left[\int_{0}^{t}\left(1-2 \mathrm{e}^{-2 \lambda \tau}+\mathrm{e}^{-4 \lambda \tau}\right) \mathrm{d} \tau\right]\left(\begin{array}{l}
1 \\
1
\end{array}\right) \\
& =t\left(1-2 \Phi_{\lambda}(t)+\Phi_{2 \lambda}(t)\right)\left(\begin{array}{l}
1 \\
1
\end{array}\right) .
\end{aligned}
$$


10 Journal of Applied Mathematics and Stochastic Analysis

Now, in view of (2.32)-(2.36), (2.31) becomes

$$
\begin{aligned}
\mathbf{s}(t)= & \lambda t\left[I+\frac{1}{2 \lambda}\left(1-\Phi_{\lambda}(t)\right) \Lambda\right]\left(\begin{array}{l}
h_{+}^{2} \\
h_{-}^{2}
\end{array}\right) \\
& +2(a+\lambda b) t\left[\left(1-\Phi_{\lambda}(t)\right) I+\frac{1}{\lambda}\left(1-(1+\lambda t) \Phi_{\lambda}(t)\right) \Lambda\right]\left(\begin{array}{c}
-h_{+} \\
h_{-}
\end{array}\right) \\
& +\frac{1}{\lambda}(a+\lambda b)^{2} t\left(1-2 \Phi_{\lambda}(t)+\Phi_{2 \lambda}(t)\right)\left(\begin{array}{l}
1 \\
1
\end{array}\right)
\end{aligned}
$$

with $\Lambda\left(\begin{array}{c}-h_{+} \\ h_{-}\end{array}\right)=\lambda\left(\begin{array}{c}h_{+}+h_{-} \\ -\left(h_{+}+h_{-}\right)\end{array}\right)=2 \lambda B\left(\begin{array}{c}1 \\ -1\end{array}\right)$ and $\Lambda\left(\begin{array}{l}h_{+}^{2} \\ h_{-}^{2}\end{array}\right)=\lambda\left(\begin{array}{c}h_{-}^{2}-h_{+}^{2} \\ h_{+}^{2}-h_{-}^{2}\end{array}\right)=4 \lambda b B\left(\begin{array}{c}-1 \\ 1\end{array}\right)$. Thus

$$
\begin{aligned}
\mathbf{s}(t)= & \lambda t\left(\begin{array}{c}
h_{+}^{2} \\
h_{-}^{2}
\end{array}\right)+2(a+\lambda b) t\left(1-\Phi_{\lambda}(t)\right)\left(\begin{array}{c}
-h_{+} \\
h_{-}
\end{array}\right) \\
& +2 B t\left[\lambda b\left(1-\Phi_{\lambda}(t)\right)-2(a+\lambda b)\left(1-(1+\lambda t) \Phi_{\lambda}(t)\right)\right]\left(\begin{array}{c}
-1 \\
1
\end{array}\right) \\
& +\frac{1}{\lambda}(a+\lambda b)^{2} t\left(1-2 \Phi_{\lambda}(t)+\Phi_{2 \lambda}(t)\right)\left(\begin{array}{l}
1 \\
1
\end{array}\right)
\end{aligned}
$$

from which we derive for instance

$$
\begin{aligned}
s_{+}(t)= & {\left[\lambda h_{+}^{2}-2(a+\lambda b) h_{+}-2 \lambda b B+4(a+\lambda b) B+\frac{1}{\lambda}(a+\lambda b)^{2}\right] t } \\
& +2\left[(a+\lambda b) h_{+}+\lambda b B-2(a+\lambda b) B(1+\lambda t)-\frac{1}{\lambda}(a+\lambda b)^{2}\right] t \Phi_{\lambda}(t) \\
& +\frac{1}{\lambda}(a+\lambda b)^{2} t \Phi_{2 \lambda}(t)
\end{aligned}
$$

Replacing $h_{+}$by $b+B$, the coefficient of $t$ in (2.39) writes

$$
\lambda(b+B)^{2}-2(a+\lambda b)(b+B)+2(2 a+\lambda b) B+\frac{a^{2}}{\lambda}+2 a b+\lambda b^{2}=\frac{a^{2}}{\lambda}+\lambda B^{2}+2(a+\lambda b) B,
$$

that of $2 t \Phi_{\lambda}(t)$ in (2.39) writes

$$
\begin{gathered}
(a+\lambda b)(b+B)-(2 a+\lambda b) B-\frac{a^{2}}{\lambda}-2 a b-\lambda b^{2}-2(a+\lambda b) B \lambda t \\
=-a\left(\frac{a}{\lambda}+B+b\right)-2(a+\lambda b) B \lambda t \\
=\gamma_{+}-2(a+\lambda b) B \lambda t .
\end{gathered}
$$

Finally, writing the term $-4(a+\lambda b) B \lambda t \Phi_{\lambda}(t)$ as $-2(a+\lambda b) B+2(a+\lambda b) B \mathrm{e}^{-2 \lambda t}$ we easily deduce (2.26) for $s_{+}$. The case of $s_{-}$is quite similar. 
Remark 2.8. If the assumption $\lambda_{+}=\lambda_{-}$is relaxed, the matrix $\Lambda$ writes $\Lambda=\left(\begin{array}{cc}-\lambda_{+} & \lambda_{+} \\ \lambda_{-} & -\lambda_{-}\end{array}\right)$and $\Lambda^{2}=-\left(\lambda_{+}+\lambda_{-}\right) \Lambda$. In this case $\mathrm{e}^{t \Lambda}=I+1 /\left(\lambda_{+}+\lambda_{-}\right)\left(1-\mathrm{e}^{-\left(\lambda_{+}+\lambda_{-}\right) t}\right) \Lambda$ and the expressions of $m_{ \pm}$and $s_{ \pm}$become much more cumbersome.

Remark 2.9. The function $m_{+}$can be directly evaluated. Indeed, since

$$
\begin{aligned}
\mathbb{E}\left(c_{\sigma_{+}(\tau)}\right) & =c_{+} \mathbb{P}\left\{N_{+}(\tau) \text { is even }\right\}+c_{-} \mathbb{P}\left\{N_{+}(\tau) \text { is odd }\right\} \\
& =\frac{1}{2} c_{+}\left(1+\mathrm{e}^{-2 \lambda \tau}\right)+\frac{1}{2} c_{-}\left(1-\mathrm{e}^{-2 \lambda \tau}\right) \\
& =A+a \mathrm{e}^{-2 \lambda \tau},
\end{aligned}
$$

we have

$$
\mathbb{E}\left(X_{+}(t)\right)=\int_{0}^{t} \mathbb{E}\left(c_{\sigma_{+}(\tau)}\right) \mathrm{d} \tau=A t+a \frac{1-\mathrm{e}^{-2 \lambda t}}{2 \lambda}=\left[A+a \Phi_{\lambda}(t)\right] t
$$

On the other hand, similarly, $\mathbb{E}\left(h_{\sigma_{+}(\tau)}\right)=B+b \mathrm{e}^{-2 \lambda \tau}$ and since the increments of $N_{+}$are independent, we have

$$
\begin{aligned}
\mathbb{E}\left(J_{+}(t)\right) & =\int_{0}^{t} \mathbb{E}\left(h_{\sigma_{+}(\tau)}\right) \mathrm{d}\left[\mathbb{E}\left(N_{+}(\tau)\right)\right] \\
& =\lambda \int_{0}^{t}\left(B+b \mathrm{e}^{-2 \lambda \tau}\right) \mathrm{d} \tau \\
& =\lambda\left[B+b \Phi_{\lambda}(t)\right] t .
\end{aligned}
$$

This confirms (2.25).

Remark 2.10. For the process $X_{+}+J_{+}$being a martingale, it is necessary (but not sufficient) for its expectation to be constant in the course of time. This condition implies, in view of (2.25), that $A+\lambda B=a+\lambda b=0$ or, equivalently, that $c_{+}+\lambda h_{+}=c_{-}+\lambda h_{-}=0$. Hence, we retrieve the conditions stated in Theorem 2.2 in the symmetric case.

It can be checked that these conditions are sufficient by computing the conditional expectation $\mathbb{E}\left[X_{+}\left(t_{2}\right)+J_{+}\left(t_{2}\right) \mid \mathbf{F}_{t_{1}}^{+}\right]$for $0 \leq t_{1} \leq t_{2}$. Indeed, we have

$$
\begin{aligned}
\mathbb{E}\left[\left(X_{+}\left(t_{2}\right)+J_{+}\left(t_{2}\right)\right)-\left(X_{+}\left(t_{1}\right)+J_{+}\left(t_{1}\right)\right) \mid \mathbf{F}_{t_{1}}^{+}\right] \\
=\mathbb{E}\left[\int_{t_{1}}^{t_{2}} c_{\sigma_{+}(\tau)} \mathrm{d} \tau+\int_{t_{1}}^{t_{2}} h_{\sigma_{+}(\tau)} \mathrm{d} N_{+}(\tau) \mid \mathbf{F}_{t_{1}}^{+}\right] \\
=\mathbb{E}\left[\int_{0}^{t_{2}-t_{1}} c_{\sigma_{+}\left(\tau+t_{1}\right)} \mathrm{d} \tau+\int_{0}^{t_{2}-t_{1}} h_{\sigma_{+}\left(\tau+t_{1}\right)} \mathrm{d} N_{+}\left(\tau+t_{1}\right) \mid \mathbf{F}_{t_{1}}^{+}\right] .
\end{aligned}
$$

Using the Markov property of the processes $\sigma_{+}$and $N_{+}$which can be stated, for $\tau \geq 0$, as

$$
\sigma_{+}\left(\tau+t_{1}\right)=\sigma_{\sigma_{+}\left(t_{1}\right)}^{\prime}(\tau), \quad N_{+}\left(\tau+t_{1}\right)=N\left(t_{1}\right)+N_{\sigma_{+}\left(t_{1}\right)}^{\prime}(\tau)
$$


12 Journal of Applied Mathematics and Stochastic Analysis

where $\sigma_{ \pm}^{\prime}$ and $N_{ \pm}^{\prime}$ are copies of the processes $\sigma_{ \pm}$and $N_{ \pm}$which are independent of $\mathbf{F}_{t_{1}}^{+}$, we obtain

$$
\begin{aligned}
\mathbb{E}\left[\left(X_{+}\left(t_{2}\right)+J_{+}\left(t_{2}\right)\right)-\left(X_{+}\left(t_{1}\right)+J_{+}\left(t_{1}\right)\right) \mid \mathbf{F}_{t_{1}}^{+}\right] \\
\quad=\mathbb{E}^{\prime}\left[\int_{0}^{t_{2}-t_{1}} c_{\sigma_{\sigma_{+}\left(t_{1}\right)}^{\prime}(\tau)} \mathrm{d} \tau+\int_{0}^{t_{2}-t_{1}} h_{\sigma_{\sigma_{+}\left(t_{1}\right)}^{\prime}(\tau)} \mathrm{d} N_{\sigma_{+}\left(t_{1}\right)}^{\prime}(\tau)\right] \\
=\mathbb{E}^{\prime}\left[X_{\sigma_{+}\left(t_{1}\right)}^{\prime}\left(t_{2}-t_{1}\right)+J_{\sigma_{+}\left(t_{1}\right)}^{\prime}\left(t_{2}-t_{1}\right)\right] \\
=m_{\sigma_{+}\left(t_{1}\right)}\left(t_{2}-t_{1}\right)=\left[A+\lambda B+\sigma_{+}\left(t_{1}\right)(a+\lambda b) \Phi_{\lambda}\left(t_{2}-t_{1}\right)\right]\left(t_{2}-t_{1}\right) .
\end{aligned}
$$

The prime superscript in $\mathbb{E}^{\prime}$ above means that the expectation applies to the functionals of the process $N^{\prime}$. So, we have $\mathbb{E}\left(X_{+}\left(t_{2}\right)+J_{+}\left(t_{2}\right) \mid \mathbf{F}_{t_{1}}\right)=X_{+}\left(t_{1}\right)+J_{+}\left(t_{1}\right)$ if and only if $A+\lambda B=$ $a+\lambda b=0$.

\section{Market model based on jump telegraph processes}

We introduce the following market model. The price of a risky asset $S(t)$ follows the equation

$$
\mathrm{d} S(t)=S(t-) \mathrm{d}\left(X_{s}(t)+J_{s}(t)\right), \quad t>0,
$$

and the process $(S(t), t \geq 0)$ is right-continuous. Here $X_{s}=\left(X_{s}(t), t \geq 0\right)$ is a telegraph process with velocity values $c_{ \pm}$, and $J_{s}=\left(J_{s}(t), t \geq 0\right)$ is a pure jump process with jump values $h_{ \pm}>-1$, which are defined in $(2.2)$, in a complete probability space $\left(\Omega, \mathscr{F}_{s}, \mathbb{P}_{s}\right)$. The initial state of the market is indicated by $s= \pm 1$.

Integrating (3.1) we obtain

$$
S(t)=S_{0} \mathscr{E}_{t}\left(X_{s}+J_{s}\right)
$$

where $S_{0}=S(0), \mathscr{E}_{t}(\cdot)$ denotes the stochastic exponential. It is easy to see that $\mathscr{E}_{t}\left(X_{s}+\right.$ $\left.J_{s}\right)=\mathrm{e}^{X_{s}(t)} \kappa_{s}(t)$, where

$$
\kappa_{s}(t)=\prod_{\tau \leq t}\left(1+\Delta J_{s}(\tau)\right)=\prod_{j=1}^{N_{s}(t)}\left(1+h_{\sigma_{s}\left(\tau_{j}-\right)}\right), \quad s= \pm .
$$

Here the $\tau_{j}, j \geq 1$, are the jumping times of the processes $N_{ \pm}$.

Indeed,

$$
\mathscr{E}_{t}(Z)=\mathrm{e}^{Z(t)-(1 / 2)\langle Z\rangle^{\text {cont }}(t)} \prod_{0<\tau \leq t}(1+\Delta Z(\tau)) \mathrm{e}^{-\Delta Z(\tau)}
$$

(see, e.g., [13, pages 77-78]). Set $Z=X_{s}+J_{s}$. As it follows from Theorem 2.2, the telegraph process without jumps cannot be a martingale. Hence $\langle X+J\rangle^{\text {cont }}=0$ and $\mathscr{E}_{t}\left(X_{s}+\right.$ $\left.J_{s}\right)=\mathrm{e}^{X_{s}(t)+J_{s}(t)} \prod_{0<\tau \leq t}\left(1+\Delta J_{s}(\tau)\right) \mathrm{e}^{-\Delta J_{s}(\tau)}=\mathrm{e}^{X_{s}(t)} \prod_{0<\tau \leq t}\left(1+\Delta J_{s}(\tau)\right)$. Therefore

$$
S(t)=S_{0} \mathrm{e}^{X_{s}(t)} \kappa_{s}(t) .
$$


On the other hand, the price of the nonrisky asset (bank account) has the form

$$
B(t)=\mathrm{e}^{Y_{s}(t)}, \quad Y_{s}(t)=\int_{0}^{t} r_{\sigma_{s}(\tau)} \mathrm{d} \tau,
$$

where $r_{+}, r_{-}>0$. Here again $Y_{s}=\left(Y_{s}(t), t \geq 0\right)$ is a telegraph process with velocity values $r_{ \pm}$.

As usual (see, e.g., [15]), we define a new probability measure $\mathbb{P}_{s}^{*}$, which is equivalent to the original measure $\mathbb{P}_{s}$. Let

$$
\frac{\mathrm{d} \mathbb{P}_{s}^{*}}{\mathrm{~d} \mathbb{P}_{s}}=\mathscr{E}_{t}\left(X_{s}^{*}+J_{s}^{*}\right)=\mathrm{e}^{X_{s}^{*}(t)} \kappa_{s}^{*}(t), \quad t \geq 0, s= \pm,
$$

be the density of the new measure with respect to measure $\mathbb{P}_{s}$. Here the jump values $h_{ \pm}^{*}$ are defined as follows: $h_{ \pm}^{*}=-c_{ \pm}^{*} / \lambda_{ \pm}>-1$. According to Theorem 2.2, for arbitrary $c_{ \pm}^{*}, c_{ \pm}^{*}<\lambda_{ \pm}$, the processes $X_{s}^{*}+J_{s}^{*}$ and $\left(\mathscr{E}_{t}\left(X_{s}^{*}+J_{s}^{*}\right), t \geq 0\right)$ are martingales.

The following theorem describes changes in the distributions with respect to the new measure (see the proof in [16]).

Theorem 3.1 (Girsanov's theorem). Under the probability measure $\mathbb{P}_{s}^{*}$,

(i) the process $N_{s}=\left(N_{s}(t), t \geq 0\right)$ is a Poisson process with alternating intensities $\lambda_{+}^{*}=$ $\lambda_{+}-c_{+}^{*}=\lambda_{+}\left(1+h_{+}^{*}\right)$ and $\lambda_{-}^{*}=\lambda_{-}-c_{-}^{*}=\lambda_{-}\left(1+h_{-}^{*}\right)$;

(ii) the process $X_{s}=\left(X_{s}(t), t \geq 0\right)$ is a telegraph process with states $\left(c_{+}, \lambda_{+}^{*}\right)$ and $\left(c_{-}, \lambda_{-}^{*}\right)$.

We now assume that the parameters of the model (3.1)-(3.6) satisfy the conditions

$$
\frac{r_{+}-c_{+}}{h_{+}}>0, \quad \frac{r_{-}-c_{-}}{h_{-}}>0
$$

Using Theorem 2.2, under such conditions we can find a unique martingale measure in the framework of the market (3.1)-(3.6). Recall that the measure $\mathbb{P}_{s}^{*}$, which is equivalent to $\mathbb{P}_{s}$, is a martingale measure if the process $\left(B(t)^{-1} S(t)\right)_{t \geq 0}$ is a $\mathbb{P}_{s}^{*}$-martingale (see, e.g., [15]). As before, $s= \pm$ indicates the initial market state.

Applying Theorem 3.1, we can construct the martingale measure for model (3.5)(3.6).

Theorem 3.2. The measure $\mathbb{P}_{s}^{*}$, defined by the density $Z_{s}(t), t \geq 0$, is the martingale measure if and only if the velocity values $c_{ \pm}^{*}$ satisfy

$$
c_{+}^{*}=\lambda_{+}-\lambda_{+}^{*}, \quad c_{-}^{*}=\lambda_{-}-\lambda_{-}^{*},
$$

where

$$
\lambda_{ \pm}^{*}=\frac{r_{ \pm}-c_{ \pm}}{h_{ \pm}}>0
$$

Moreover, under the probability measure $\mathbb{P}_{s}^{*}$, the process $N_{s}$ is a Poisson process with alternating intensities $\lambda_{ \pm}^{*}$.

Proof. According to Theorem 3.1, the process $X_{s}-Y_{s}$ is a telegraph process (with respect to $\mathbb{P}_{s}^{*}$ ) with the velocity values $c_{ \pm}-r_{ \pm}$and the alternating intensities $\lambda_{ \pm}^{*}=\lambda_{ \pm}-c_{ \pm}^{*}$. From 
14 Journal of Applied Mathematics and Stochastic Analysis

Theorem 2.2, it follows that $\left(X_{s}(t)-Y_{s}(t)+J_{s}(t), t \geq 0\right)$ is the $\mathbb{P}_{s}^{*}$-martingale if and only if

$$
\left(\lambda_{+}-c_{+}^{*}\right) h_{+}=-\left(c_{+}-r_{+}\right), \quad\left(\lambda_{-}-c_{-}^{*}\right) h_{-}=-\left(c_{-}-r_{-}\right) .
$$

Hence $c_{ \pm}^{*}=\lambda_{ \pm}+\left(c_{ \pm}-r_{ \pm}\right) / h_{ \pm}$and $h_{ \pm}^{*}=-c_{ \pm}^{*} / \lambda_{ \pm}=-1+\left(r_{ \pm}-c_{ \pm}\right) /\left(\lambda_{ \pm} h_{ \pm}\right)>-1$, and the theorem is proved.

In the framework of this model, option pricing formulae and hedging strategies are completely constructed (see $[17,16]$ ). The arbitrage-free price $c$ of a call option with expiry payoff $(S(T)-K)^{+}$can be calculated by the formula

$$
c=c_{s}=S_{0} u_{s}\left(y, T ; \bar{\lambda}_{ \pm}, 0\right)-K u_{s}\left(y, T ; \lambda_{ \pm}^{*}, r_{ \pm}\right), \quad s= \pm,
$$

where $\bar{\lambda}_{ \pm}=\lambda_{ \pm}^{*}\left(1+h_{ \pm}\right), \lambda_{ \pm}^{*}=\left(r_{ \pm}-c_{ \pm}\right) / h_{ \pm}>0, y=\ln K / S_{0}$.

For giving a representation of functions $u_{ \pm}$, we need several settings. Let us introduce

$$
\rho_{ \pm}^{(n)}(t)=\mathrm{e}^{-\left(\lambda_{-}+r_{-}\right) t} \Lambda_{ \pm}^{(n)} P_{ \pm}^{(n)}(t)
$$

with $\Lambda_{+}^{(n)}=\left(\lambda_{+}\right)^{[(n+1) / 2]}\left(\lambda_{-}\right)^{[n / 2]}, \Lambda_{-}^{(n)}=\left(\lambda_{-}\right)^{[(n+1) / 2]}\left(\lambda_{+}\right)^{[n / 2]}$, and

$$
\begin{aligned}
P_{ \pm}^{(n)}(t) & =\frac{t^{n}}{n !} \cdot{ }_{1} F_{1}\left(m_{n}^{( \pm)}+1 ; n+1 ;-\delta t\right), m_{n}^{(+)} \\
& =[n / 2], m_{n}^{(-)} \\
& =[(n-1) / 2], \delta=\lambda_{+}-\lambda_{-}+r_{+}-r_{-} .
\end{aligned}
$$

Recall that the usual hypergeometric function ${ }_{1} F_{1}(\alpha ; \beta ; z)$ is defined as (see [18])

$$
{ }_{1} F_{1}(\alpha ; \beta ; z)=1+\sum_{n=1}^{\infty} \frac{\alpha(\alpha+1) \cdots(\alpha+n-1)}{n ! \beta(\beta+1) \cdots(\beta+n-1)} z^{n}=1+\sum_{n=1}^{\infty} \frac{(\alpha)_{n}}{n !(\beta)_{n}} z^{n} .
$$

Notice that $P_{+}^{(2 n+1)} \equiv P_{-}^{(2 n+1)}:=P^{(2 n+1)}, n=0,1,2, \ldots$ Let us also introduce $w_{ \pm}^{(n)}(p, q)=$ $\mathrm{e}^{-\left(\lambda_{+}+r_{+}\right) q-\left(\lambda_{-}+r_{-}\right) p} \Lambda_{ \pm}^{(n)} v_{ \pm}^{(n)}(p, q)$, where for positive $p$, $q$, we define $v_{-}^{(0)}(p, q) \equiv 0, v_{+}^{(0)}(p, q)$ $=\mathrm{e}^{-\delta p}, v_{ \pm}^{(1)}(p, q)=P^{(1)}(p)$, and for $n \geq 1$

$$
\begin{aligned}
v_{ \pm}^{(2 n+1)}(p, q) & =P^{(2 n+1)}(p)+\sum_{k=1}^{n} \frac{q^{k}}{k !} \sum_{j=0}^{k-1} \delta^{k-j-1} \beta_{k, j} P_{-}^{(2 n-j)}(p), \\
v_{+}^{(2 n)}(p, q) & =P_{+}^{(2 n)}(p)+P^{(2 n-1)}(p)+\sum_{k=2}^{n} \frac{q^{k}}{k !} \sum_{j=0}^{k-2} \delta^{k-j-2} \beta_{k-1, j} P_{-}^{(2 n-j-2)}(p), \\
v_{-}^{(2 n)}(p, q) & =P_{-}^{(2 n)}(p)+\sum_{k=1}^{n-1} \frac{q^{k}}{k !} \sum_{j=0}^{k} \delta^{k-j} \beta_{k+1, j} P_{-}^{(2 n-j)}(p) .
\end{aligned}
$$

In (3.16) the coefficients $\beta_{k, j}, j<k$, are defined as follows: $\beta_{k, 0}=\beta_{k, 1}=\beta_{k, k-2}=\beta_{k, k-1}=1$,

$$
\beta_{k, j}=\frac{(k-j)_{[j / 2]}}{[j / 2] !} .
$$


With these settings on hand, functions $u_{ \pm}$can be expressed as

$$
u_{s}\left(y, T ; \lambda_{ \pm}\right)=\sum_{n=0}^{\infty} u_{s}^{(n)}\left(y-b_{s}^{(n)}, T ; \lambda_{ \pm}, r_{ \pm}\right), \quad b_{ \pm}^{(n)}=\sum_{j=0}^{n} \ln \left(1+h_{\sigma_{ \pm}\left(\tau_{j}-\right)}\right)
$$

where $u_{ \pm}^{(n)}$ is given by

$$
u_{ \pm}^{(n)}(y, t)= \begin{cases}0, & y>c_{+} t \\ w_{ \pm}^{(n)}\left(\frac{c_{+} t-y}{2 a}, \frac{y-c_{-} t}{2 a}\right), & c_{-} t \leq y \leq c_{+} t, \quad n \geq 0 \\ \rho_{ \pm}^{(n)}(t), & y<c_{-} t\end{cases}
$$

For the detailed proof of (3.12)-(3.16) (if $r_{+}=r_{-}$) see [17].

It is interesting to analyze the problem of convergence to the famous Black-Scholes model. First of all, note that the symmetric telegraph process associated with the parameters $\lambda_{+}=\lambda_{-}:=\lambda, c_{+}=a, c_{-}=-a$ converges to the standard Brownian motion $(w(t), t \geq$ 0 ) if $a, \lambda \rightarrow \infty$, such that $a^{2} / \lambda \rightarrow 1$ (see [12] or [19]).

The following theorem provides a similar connection under respective scaling between market model driven by geometric telegraph processes (with jumps) and geometric Brownian motion.

Suppose that $a, \lambda_{-}, \lambda_{+} \rightarrow \infty$, and $h_{-}, h_{+} \rightarrow 0$ such that

$$
\frac{a^{2}}{\lambda_{ \pm}} \longrightarrow \sigma^{2}, \quad \sqrt{\lambda_{ \pm}} h_{ \pm} \longrightarrow \alpha_{ \pm} .
$$

Although the jump telegraph process is of finite variation and of finite activity around the origin, this scaling agrees with empirical observations of Carr et al. [2].

Theorem 3.3. Under scaling (3.20), assume that the following limit exists:

$$
A+\frac{\lambda_{+}}{2} \ln \left(1+h_{+}\right)+\frac{\lambda_{-}}{2} \ln \left(1+h_{-}\right) \longrightarrow \mu
$$

Then model (3.2) converges in distribution to the Black-Scholes model:

$$
S(t) \stackrel{D}{\longrightarrow} S_{0} \mathrm{e}^{v w(t)+\mu t}
$$

where $\stackrel{D}{\rightarrow}$ denotes convergence in distribution, and $v^{2}=\left(\sigma+\left(\alpha_{+}-\alpha_{-}\right) / 2\right)^{2}+\left(\alpha_{+}^{2}+\alpha_{-}^{2}\right) / 2$.

Proof. Let $f_{ \pm}(z, t)=\mathbb{E}\left[\mathrm{e}^{z X_{ \pm}(t)} \kappa_{ \pm}(t)^{z}\right]$ be the moment-generating function of $\ln S(t)=$ $X_{ \pm}(t)+\ln \kappa_{ \pm}(t)$. We prove here the convergence

$$
f_{ \pm}(z, t) \longrightarrow \exp \left(\mu z t+\frac{v^{2} z^{2} t}{2}\right)
$$

which is sufficient for the convergence of pointwise distributions in (3.22). 
16 Journal of Applied Mathematics and Stochastic Analysis

Recalling that $X_{ \pm}(t)=A t+a X_{ \pm}^{0}(t)$, where $X_{ \pm}^{0}(t)=\int_{0}^{t} \sigma_{ \pm}(\tau) \mathrm{d} \tau$, we get

$$
f_{ \pm}(z, t)=\mathbb{E}\left[\mathrm{e}^{z\left(A t+a X_{ \pm}^{0}(t)\right)} \kappa_{ \pm}(t)^{z}\right]=\mathrm{e}^{A z t-\lambda t} \sum_{n=0}^{\infty} \int_{-\infty}^{\infty} \mathrm{e}^{a z x-\Lambda x} \kappa_{ \pm}^{(n)} q_{ \pm}^{(n)}\left(x-j_{ \pm}^{(n)}, t\right) \mathrm{d} x
$$

where $\lambda=\left(\lambda_{+}-\lambda_{-}\right) / 2, \Lambda=\left(\lambda_{+}+\lambda_{-}\right) / 2$ and $\kappa_{ \pm}^{(n)}$ are defined as in (2.19) with $\left(1+h_{ \pm}\right)^{z}$ instead of $\lambda_{ \pm}^{\prime} / \lambda_{ \pm}$(and with $\left.a=1\right)$.

Let $\bar{p}_{ \pm}^{0}$ be the (generalized) probability densities of the standard telegraph processes $\bar{X}_{ \pm}^{0}$, which are controlled by the Poisson process with alternating intensities $\bar{\lambda}_{ \pm}=\lambda_{ \pm}(1+$ $\left.h_{ \pm}\right)^{z}$, and set $\bar{\Lambda}=\left(\bar{\lambda}_{+}+\bar{\lambda}_{-}\right) / 2, \Lambda=\left(\lambda_{+}+\lambda_{-}\right) / 2, \bar{\lambda}=\left(\bar{\lambda}_{+}-\bar{\lambda}_{-}\right) / 2, \lambda=\left(\lambda_{+}-\lambda_{-}\right) / 2$.

Using (2.14)-(2.17) (see also (2.18) and (2.19)), one can deduce

$$
\begin{aligned}
f_{ \pm}(z, t) & =\mathrm{e}^{A z t-\Lambda t} \sum_{n=0}^{\infty} \int_{-\infty}^{\infty} \mathrm{e}^{a z x-\lambda x}{q^{\prime}}_{ \pm}^{(n)}\left(x-j_{ \pm}^{(n)}, t\right) \mathrm{d} x \\
& =\mathrm{e}^{A z t+(\bar{\Lambda}-\Lambda) t} \int_{-\infty}^{\infty} \mathrm{e}^{a z x+(\bar{\lambda}-\lambda) x} \bar{p}_{ \pm}^{0}(x, t) \mathrm{d} x \\
& =\frac{1}{a} \mathrm{e}^{A t z+(\bar{\Lambda}-\Lambda) t} \int_{-\infty}^{\infty} \mathrm{e}^{(z+(\bar{\lambda}-\lambda) / a) x} \bar{p}_{ \pm}^{0}\left(\frac{x}{a}, t\right) \mathrm{d} x .
\end{aligned}
$$

Note that under scaling $a^{2} / \lambda_{ \pm} \rightarrow \sigma^{2}$ we have the convergence to normal distribution $\mathcal{N}\left(0, t \sigma^{2}\right)$ :

$$
\frac{1}{a} \bar{p}_{ \pm}^{0}\left(\frac{x}{a}, t\right) \longrightarrow \frac{1}{\sigma \sqrt{2 \pi t}} \mathrm{e}^{-x^{2} /\left(2 \sigma^{2} t\right)}
$$

Then, it follows from (3.21) that

$$
\begin{aligned}
A t z+(\bar{\Lambda}-\Lambda) t= & A t z+t\left(\frac{\lambda_{+}}{2}\left[\left(1+h_{+}\right)^{z}-1\right]+\frac{\lambda_{-}}{2}\left[\left(1+h_{-}\right)^{z}-1\right]\right) \\
= & t z\left(A+\frac{\lambda_{+}}{2} \ln \left(1+h_{+}\right)+\frac{\lambda_{-}}{2} \ln \left(1+h_{-}\right)\right) \\
& +\frac{t z^{2}}{4}\left[\lambda_{+} \ln \left(1+h_{+}\right)^{2}+\lambda_{-} \ln \left(1+h_{-}\right)^{2}\right]+o(1) \longrightarrow \mu t z+\frac{\alpha_{+}^{2}+\alpha_{-}^{2}}{4} t z^{2}
\end{aligned}
$$

and one can derive from (3.20)

$$
\begin{aligned}
\frac{\bar{\lambda}-\lambda}{a} & =\frac{\lambda_{+}}{2 a}\left[\left(1+h_{+}\right)^{z}-1\right]-\frac{\lambda_{-}}{2 a}\left[\left(1+h_{-}\right)^{z}-1\right] \\
& \sim z \frac{\lambda_{+} h_{+}-\lambda_{-} h_{-}}{2 a} \longrightarrow z \frac{\alpha_{+}-\alpha_{-}}{2 \sigma} .
\end{aligned}
$$

Summarizing the above statements, we obtain the convergence (3.23).

Remark 3.4. Condition (3.21) in this theorem means that the total drift $A+\left[\lambda_{-} \ln (1+\right.$ $\left.\left.h_{-}\right)+\lambda_{+} \ln \left(1+h_{+}\right)\right] / 2$ is asymptotically finite. Here $A=\left(c_{+}+c_{-}\right) / 2$ is generated by the 
velocities of telegraph process $X$, and the summand $\left[\lambda_{-} \ln \left(1+h_{-}\right)+\lambda_{+} \ln \left(1+h_{+}\right)\right] / 2$ represents the drift component (possibly with infinite asymptotics) that is motivated only by jumps. If in (3.21) the limits of $\lambda_{ \pm} \ln \left(1+h_{ \pm}\right)$are finite, then $A \rightarrow$ const, and $\alpha_{+}=\alpha_{-}=0$. In this case, the volatility of the limit is $v^{2}=\lim a^{2} / \lambda_{ \pm}$.

Hence in model (3.1)-(3.6) values $a / \sqrt{\lambda_{ \pm}}$can be interpreted as "telegraph" components of the volatility, and $\sqrt{\lambda_{ \pm}} \ln \left(1+h_{ \pm}\right)$are the volatilities engendered by jumps.

The limiting volatility $v=\sqrt{\left(\sigma+\left(\alpha_{+}-\alpha_{-}\right) / 2\right)^{2}+\left(\alpha_{+}^{2}+\alpha_{-}^{2}\right) / 2}$ depends both on "telegraph" and jump components. The dependence of the jump components $\alpha_{ \pm}$has a character of a "volatility smile" with minimum value $v_{\min }^{2}=\sigma^{2} / 2$ at $\alpha_{+}=-\sigma / 2, \alpha_{-}=\sigma / 2$.

\section{Memory effects and historical volatility in the framework of jump telegraph market model}

In this section, we explain how the simple model (3.1) can capture memory effects. Let us define the historical volatility

$$
\mathrm{HV}(t-\tau)=\sqrt{\frac{\operatorname{Var}\{\ln (S(t) / S(\tau))\}}{t-\tau}}, \quad t>\tau \geq 0 .
$$

For models without memory the historical volatility coincides with the implied volatility. For example, the Black-Scholes model has $\operatorname{HV}(t) \equiv \sigma$. It is interesting to describe the behavior of $\mathrm{HV}(t)$ in our model (3.1).

We introduce the notation $\operatorname{HV}_{ \pm}(t)=f_{ \pm}(t)$, where function $f_{ \pm}$is given by $f_{ \pm}(t)=$ $\sqrt{s_{ \pm}(t) / t}$. Here $s_{ \pm}(t)=\operatorname{Var}\left(X_{ \pm}(t)+J_{ \pm}(t)\right)$. The exact formula for $s_{ \pm}$is presented by $(2.26)$ with $\ln \left(1+h_{ \pm}\right)$instead of $h_{ \pm}$. Hence

$$
f_{ \pm}(t)=\sqrt{a^{2} / \lambda+\lambda B^{2}+(a+\lambda b)^{2} \Phi_{2 \lambda}(t) / \lambda+\gamma_{ \pm} \Phi_{\lambda}(t) \pm 2 B(a+\lambda b) \mathrm{e}^{-2 \lambda t}}
$$

It is interesting to compare model (3.1) with moving-average type models, which are sometimes explored to capture memory effects. The simplest form of such model is

$$
\ln \left(\frac{S(t)}{S(0)}\right)=a t+\sigma w(t)-\sigma \int_{0}^{t} \mathrm{~d} \tau \int_{-\infty}^{\tau} p \mathrm{e}^{-(q+p)(\tau-u)} \mathrm{d} w(u)
$$

where $\sigma, q, p+q>0$ and $w=(w(t), t \geq 0)$ is a standard Brownian motion (see [20, examples 2.12 and 2.14] or [21]). In this case, the historical volatility is described by

$$
f(t)=\frac{\sigma}{2 \lambda} \sqrt{q^{2}+p(2 q+p) \Phi_{\lambda}(t)}
$$

with $2 \lambda=p+q$. Expressions (4.2) and (4.4) look similar.

Notice that

$$
\begin{aligned}
& \lim _{t \rightarrow+0} f_{ \pm}(t)=\sqrt{\lambda}\left|\ln \left(1+h_{ \pm}\right)\right| \\
& \lim _{t \rightarrow+\infty} f_{ \pm}(t)=\sqrt{a^{2} / \lambda+\lambda\left(\left[\ln \left(1+h_{+}\right)\left(1+h_{-}\right)\right] / 2\right)^{2}} .
\end{aligned}
$$


These limits look reasonable: the limit at 0 is engendered only by jumps, the limit at $\infty$ contains both the "telegraph" component and a long-term influence of jumps. By the way, in the case of minimum influence of jumps on volatility, $\ln \left(1+h_{+}\right)\left(1+h_{-}\right)=0$ (and $\alpha_{+}=-\sigma / 2, \alpha_{-}=\sigma / 2$, see Remark 3.4), the long-term limit contains only the "telegraph" component: $\lim _{t \rightarrow \infty} f_{ \pm}(t)=a / \sqrt{\lambda}$.

\section{Acknowledgments}

The author thanks the referees for a very careful reading of the manuscript and many helpful remarks and suggestions.

\section{References}

[1] T. Björk and H. Hult, "A note on Wick products and the fractional Black-Scholes model," Finance and Stochastics, vol. 9, no. 2, pp. 197-209, 2005.

[2] P. Carr, H. German, D. B. Madan, and M. Yor, "The fine structure of asset returns: an empirical investigation," The Journal of Business, vol. 75, no. 2, pp. 305-332, 2002.

[3] P. Carr, H. Geman, D. B. Madan, and M. Yor, "Pricing options on realized variance," Finance and Stochastics, vol. 9, no. 4, pp. 453-475, 2005.

[4] R. J. Elliott and C.-J. U. Osakwe, "Option pricing for pure jump processes with Markov switching compensators," Finance and Stochastics, vol. 10, no. 2, pp. 250-275, 2006.

[5] S. Goldstein, "On diffusion by discontinuous movements and on the telegraph equation," The Quarterly Journal of Mechanics and Applied Mathematics, vol. 4, no. 2, pp. 129-156, 1951.

[6] M. Kac, "A stochastic model related to the telegrapher's equation," The Rocky Mountain Journal of Mathematics, vol. 4, pp. 497-509, 1974.

[7] E. Orsingher, "Probability law, flow function, maximum distribution of wave-governed random motions and their connections with Kirchoff's laws," Stochastic Processes and their Applications, vol. 34, no. 1, pp. 49-66, 1990.

[8] S. Zacks, "Generalized integrated telegraph processes and the distribution of related stopping times," Journal of Applied Probability, vol. 41, no. 2, pp. 497-507, 2004.

[9] G. B. Di Mazi, Y. M. Kabanov, and V. I. Runggal'der, "Mean-variance hedging of options on stocks with Markov volatilities," Theory of Probability and Its Applications, vol. 39, no. 1, pp. 172-182, 1994.

[10] C. Mazza and D. Rullière, "A link between wave governed random motions and ruin processes," Insurance: Mathematics \& Economics, vol. 35, no. 2, pp. 205-222, 2004.

[11] A. Di Crescenzo and F. Pellerey, "On prices' evolutions based on geometric telegrapher's process," Applied Stochastic Models in Business and Industry, vol. 18, no. 2, pp. 171-184, 2002.

[12] M. Kac, Probability and Related Topics in Physical Sciences, vol. 1 of Lectures in Applied Mathematics, Interscience Publishers, London, UK, 1959.

[13] P. Protter, Stochastic Integration and Differential Equations. A New Approach, vol. 21 of Applications of Mathematics, Springer, Berlin, Germany, 1990.

[14] L. Beghin, L. Nieddu, and E. Orsingher, "Probabilistic analysis of the telegrapher's process with drift by means of relativistic transformations," Journal of Applied Mathematics and Stochastic Analysis, vol. 14, no. 1, pp. 11-25, 2001.

[15] I. Karatzas and S. E. Shreve, Methods of Mathematical Finance, vol. 39 of Applications of Mathematics, Springer, New York, NY, USA, 1998.

[16] N. Ratanov, “A jump telegraph model for option pricing," Quantitative Finance, vol. 7, no. 5, pp. 575-583, 2007.

[17] N. Ratanov, "Pricing options under telegraph processes," Revista de Economia del Rosario, vol. 8, no. 2, pp. 131-150, 2005. 
[18] M. Abramowitz and I. A. Stegun, Handbook of Mathematical Functions with Formulas, Graphs, and Mathematical Tables, Dover, New York, NY, USA, 1972.

[19] N. Ratanov, "Telegraph evolutions in inhomogeneous media," Markov Processes and Related Fields, vol. 5, no. 1, pp. 53-68, 1999.

[20] V. Anh and A. Inoue, "Financial markets with memory. I. Dynamic models," Stochastic Analysis and Applications, vol. 23, no. 2, pp. 275-300, 2005.

[21] A. Inoue, Y. Nakano, and V. Anh, "Linear filtering of systems with memory and application to finance," Journal of Applied Mathematics and Stochastic Analysis, vol. 2006, Article ID 53104, 26 pages, 2006.

Nikita Ratanov: Faculty of Economics, Universidad del Rosario, Calle 14, No.4-69,

Bogotá, Colombia

Email address: nratanov@urosario.edu.co 


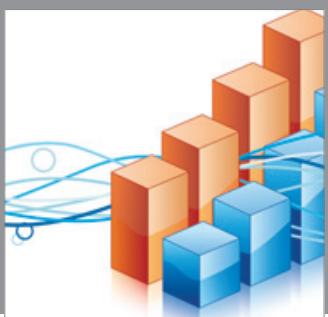

Advances in

Operations Research

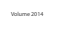

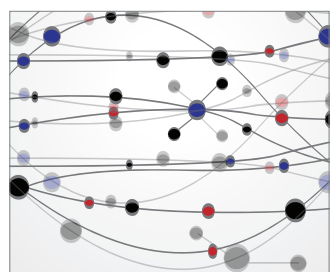

\section{The Scientific} World Journal
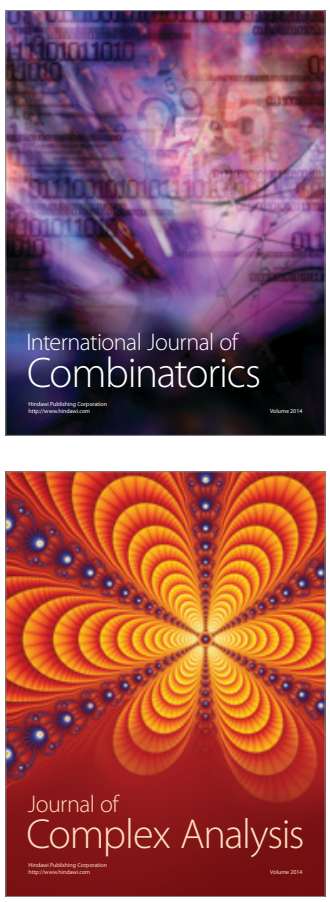

International Journal of

Mathematics and

Mathematical

Sciences
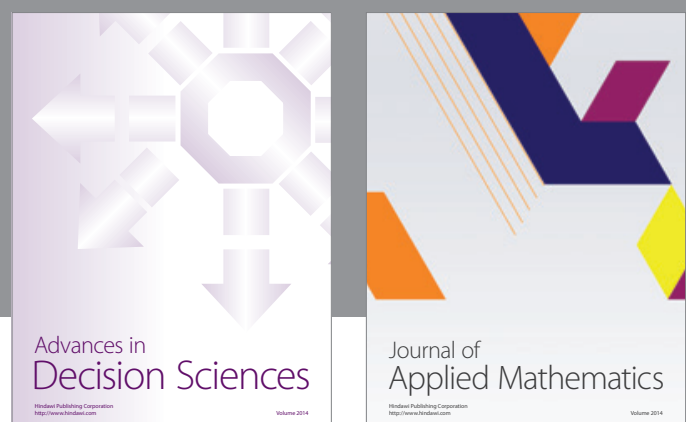

Journal of

Applied Mathematics
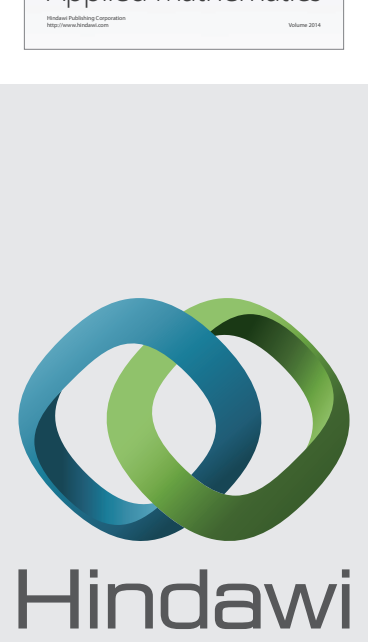

Submit your manuscripts at http://www.hindawi.com
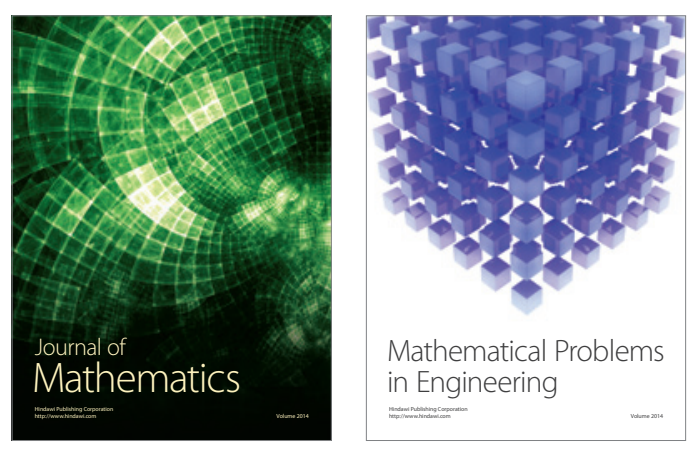

Mathematical Problems in Engineering
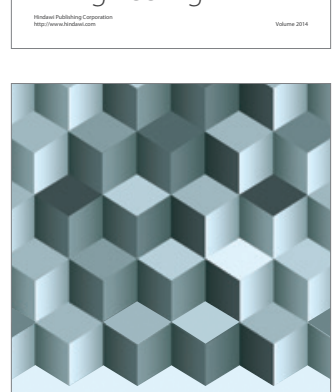

Journal of

Function Spaces
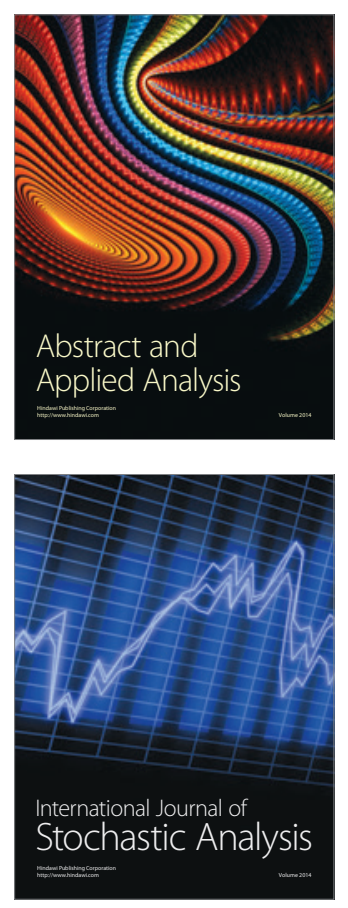

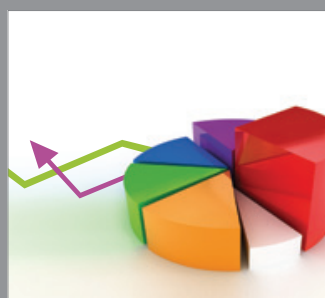

ournal of

Probability and Statistics

Promensencen
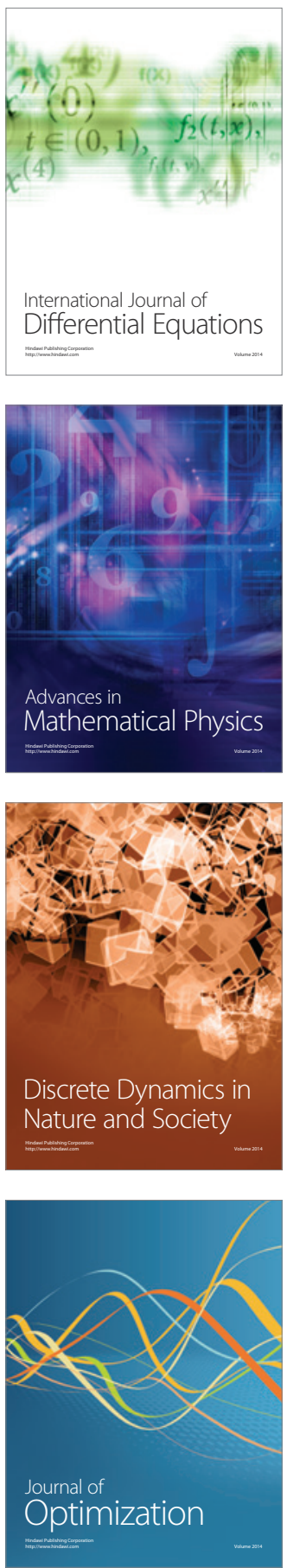